\title{
The plant as pugilist
}

\section{Ian T. Baldwin revels in a volume that reminds us of the arsenals of the undergrowth.}

$\mathrm{T}$ The view of plants as passive organisms that consume sunlight, produce oxygen and create soothing sensory environments is all down to our ancestors' domestication of a handful of wild species. In fact, the untamed native plant is a pugilist: feeding others is not its raison d'être. Defensive toxins such as cyanide and mustard oil lace the tissues of many plants.

Dale Walters's Fortress Plant celebrates how wild plants thrive at the base of the food chain. This lively narrative of "how to survive when everything wants to eat you" (as the subtitle has it) weaves important new research and perspective into the structure of Walters's successful 2010 Plant Defense (Wiley). His list of plants' foes is refreshingly comprehensive. There are the microscopic: the pathogenic bacteria, fungi and Oomycetes (a distinct lineage that masquerades as fungi). There are the macroscopic, such as insects, mammals and birds. And there are the plants that have strayed from their photosynthetic ways and turned to the dark side - the parasites, such as Cuscuta, or dodder.

Walters's list bridges a chasm. For some three decades, research on resistance traits at the two spatial scales of attackers (pathogens and herbivores) has been driven by two broad groups with different training and skills, who publish their findings in different journals and have not communicated productively. Each has focused on different parts of the fight. Evolutionary biologists, frequently trained in entomology and small-molecule chemistry, formed the plant-herbivore community. They uncovered resistance traits, the diverse chemical defences against predation. Botanists with microbial training, meanwhile, studied plant-pathogen interactions, and focused on the early signalling events that allow plants to recognize and respond to attack.

Walters's comfort zone is clearly the realm of the smallest attackers, and how plants thwart them at the cellular and subcellular scales. Signalling mechanisms, such as molecules that recognize attack, discern the panoply of microbial bad guys. These include biotrophic and necrotrophic pathogens that attack living and dead plants, respectively, and 'change artists' that enter the plant as biotrophs and transform into necrotrophs. Much work is needed to understand how wild plants' signalling systems distinguish these from 'good guys' such as endophytic fungi and bacteria, symbionts recruited by

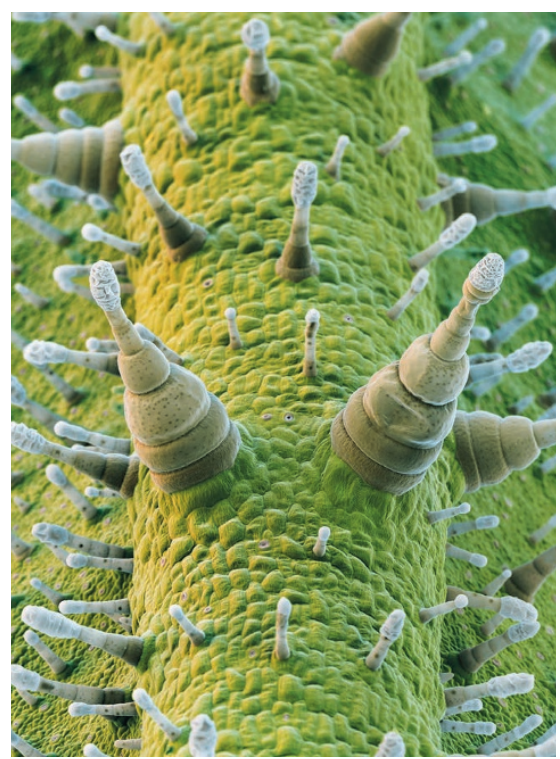

The protective glandular hairs of coyote tobacco.

the plant to solve problems such as poorquality soils or attack by enemies.

Fortress Plant also admirably tackles plant tricks that work against larger herbivores. Here, the theatre of engagement can extend to the neighbouring plant community. Signals that activate resistance traits, such as antidigestive protease inhibitors, can be wafted on the wind or transmitted through the underground mycorrhizal networks, built by fungi, that connect plants. Traits including volatile alarm calls lure helpers such as insect predators to the attacked plant. Thus, by attracting carnivores, the plant rids itself of the vegetarians. This 'enemy-of-my-enemy-ismy-friend' strategy works brilliantly against insects attackers that have their own mobile foes. All attacked plants need do is produce signals that betray the vegetarians' position.

The book's disciplinary bridging is notable in passages on advances that each faction has made on the other's turf. The plantherbivore community has uncovered the early signalling events that allow a wild plant to recognize attack from herbivores. The plant-pathogen community has revealed the traits that underlie pathogen resistance.

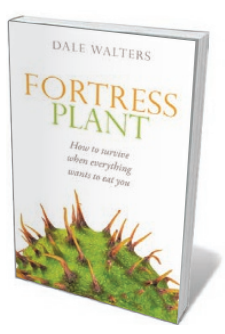

Fortress Plant: How to Survive When Everything Wants to Eat You DALE WALTERS Oxford University Press: 2017.
Walters's personal reflections on the scientists who inspired his own three decades of research into plant pathology help him create a unified narrative. Key is the pioneering work of Joseph Kuć and his colleagues at the University of Kentucky in Lexington, who characterized whole-plant responses to localized pathogen challenges.

A pervasive issue is Walters's use of military metaphors. Although they are inevitable in this context, Walters often over-eggs: the chapter titles, for instance, range from "Call to arms" to "Weapons of war". Such language has shaped the mental constructs of biologists who study "evolutionary arms races". But military metaphors themselves evolve as wars shift from outright destruction to the gain of strategic aims, and the military rarely leads when it comes to understanding interactions. Plant-heterotroph interplay is likely to be nuanced, given how long most of the partners have been at it. Summoning ideas of conflict can limit thinking, as doctors have found in treating disease.

Thus the plant-herbivore community has been careful to reserve 'defence' for traits that increase a plant's Darwinian fitness in environments with attackers. 'Tolerance' traits are those that reduce the harm of attack without damaging the attacker. For example, a plant might send a pest to attack old leaves rather than young ones, benefitting both the plant and the pathogen.

Tolerance traits - such as the kinase enzymes that place plant sugars in belowground storage during attack - are not mentioned in Walters's narrative. Most researchers who use laboratory bioassays to study these interactions have little to say about such traits, which must be analysed in the field. The caterpillar or tobacco hornworm Manduca sexta, for example, is the main pest of the plant I work on (coyote tobacco, Nicotiana attenuata, which grows across swathes of the western United States). Yet the adult, the Carolina sphinx moth, is the plant's preferred pollinator. So an overdefended fortress will not enjoy the evolutionary benefits of 'outcrossing. There is a lesson in that for plant researchers as we find common ground in analysing the traits that allow flora to produce successful grandchildren in risky environments.

Ian T. Baldwin is at the Max Planck Institute for Chemical Ecology, Jena, Germany. e-mail:baldwin@ice.mpg.de 